\title{
Correction to: Rice Production, Augmentation, Escalation, and Yield Under Water Stress
}

\author{
U. Maalik, M. Farid, M. Zubair, S. Ali, M. Rizwan, M. Shafqat, \\ and H. K. Ishaq
}

\section{Correction to:}

Chapter 7 in: M. Hasanuzzaman (ed.),

\section{Agronomic Crops,}

https://doi.org/10.1007/978-981-15-0025-1_7

The book was inadvertently published with an incorrect spelling of the author's name in Chapter 7 as M. Riwan whereas it should be M. Rizwan. 